\title{
Herramientas de aprendizaje para favorecer la educación ambiental. Caso de estudio Fundación Niños de Los Andes sede Manizales, Colombia
}

\author{
Learning Tools in Environmental Education at \\ Fundación Niños de Los Andes in Manizales, Colombia
}

\begin{abstract}
Ferramentas de aprendizagem para promover a educação ambiental. Estudo de caso da Fundação Criança de Los Andes, Manizales, Colômbia
\end{abstract}

\begin{abstract}
Gloria Yaneth Flórez-Yepes ${ }^{1}$
Universidad Católica de Manizales

Manizales, Colombia

gyflorez@ucm.edu.co

Alejandro Rincón-Santamaría

Universidad Católica de Manizales

Manizales, Colombia

arincons@ucm.edu.co

Pablo Santiago Cardona ${ }^{3}$

Universidad Católica de Manizales

Manizales, Colombia

pandora@ucm.edu.co

Felipe Antonio Gallego 4

Universidad Católica de Manizales

Manizales, Colombia

fagallego@ucm.edu.co

Recibido • Received • Recebido: 20 / 06 / 2016

Corregido • Revised • Revisado: 29 / 01 / 2018

Aceptado • Accepted • Aprovado: 13/ 02 / 2018

\footnotetext{
${ }^{1}$ Administradora ambiental y de recursos naturales, Magister en desarrollo sostenible y medio ambiente, Docente investigadora Universidad Católica de Manizales.

${ }^{2}$ Ingeniero químico, Magister en Ingeniería, Doctor en Ingeniería, Docente Investigador Universidad Católica de Manizales.

${ }^{3}$ Arquitecto, maestrante en Maestría en Educación, Docente Investigador Universidad Católica de Manizales.

${ }^{4}$ Matemático, magister en ciencias Matemática Aplicada, docente investigador Universidad Católica de Manizales, asesor estadístico de la Dirección de Investigaciones y Posgrados (Universidad Católica de Manizales).
} 
doi: http://dx.doi.org/10.15359/ree.22-2.5

URL: http://www.una.ac.cr/educare

CORREO: educare@una.cr

Resumen: Este artículo es parte de los resultados de un proyecto de investigación donde el objetivo es formular una estrategia de sensibilización ambiental para niños, niñas y adolescentes de la Fundación Niños de Los Andes en la ciudad de Manizales. El proceso metodológico se aborda con un enfoque mixto y de tipo descriptivo, utilizando la técnica de la encuesta y como instrumento de recolección de información el cuestionario y cuya herramienta de sistematización fue el software SPSS. Se analiza el contexto tanto desde el estudiantado como desde las posturas del personal docente, igualmente para el diseño metodológico se trabaja con el $100 \%$ de estudiantes desde los grados 3 a 11. Posteriormente se realizó la discusión de los resultados y se definió cuál es la mejor forma de abordaje de la educación ambiental en la niñez. El principal resultado muestra que la mejor herramienta para la aceptación de la educación ambiental en la niñez se implemente a partir del desarrollo de ejercicios prácticos que les permitan conocer a través de casos reales las problemáticas ambientales y sus formas de abordaje para prevenirlas, mitigarlas y controlarlas.

Palabras claves: Educación ambiental; estrategia de sensibilización; herramientas de aprendizaje; manejo de residuos.

Abstract: This article is part of the results of a research project whose objective was to formulate a strategy for environmental awareness for children and adolescents of the Fundación Niños de Los Andes (Children of the Andes Foundation) in the city of Manizales, Colombia. The methodological process was a quantitative and descriptive approach, using the survey as a tool for information gathering and the SPSS tool for systematization. A later discussion of the results was performed, and it determined the best way to approach the environmental education in children. The main conclusion was that the best strategy for acceptance of environmental education in children is to develop practical workshops that enable them to know, through real cases, environmental issues and ways of approach to prevent these issues, mitigate them, and control them.

Keywords: Environmental education; awareness strategy; wastewater treatment; waste management.

Resumo: Este artigo faz parte dos resultados de um projeto de pesquisa onde o objetivo é formular uma estratégia de consciência ambiental para crianças e adolescentes da Fundação Crianças de Los Andes, na cidade de Manizales, Colômbia. A metodologia é abordada desde um enfoque misto e descritivo, utilizando a técnica de enquete e um questionário como instrumento para coletar as informação, no qual foi utilizado a ferramenta software SPSS para sistematizar os dados. O contexto, tanto dos estudantes como também dos professores, faz parte da análise para desenhar a metodologia do projeto, que foi elaborada com $100 \%$ dos estudantes de $3^{\circ}$ a $11^{\circ} \mathrm{grau}$. Posteriormente, foi feita a discussão dos resultados e foi definida qual seria a melhor maneira de abordar a educação ambiental na infância. O principal resultado mostra que a melhor ferramenta para a aceitação da educação ambiental na infância se dá através do desenvolvimento de exercícios práticos, que lhes permitam aprender através de casos reais, questões e problemas ambientais e formas de abordagem de prevenção, mitigação e controle.

Palavras-chave: Educação ambiental; estratégia de sensibilização; ferramentas de aprendizagem; gestão de resíduos. 


\section{Introducción}

La educación ambiental en Colombia, al igual que los medios y las herramientas que se han utilizado para fomentarla, ha contribuido de forma significativa en la construcción e interiorización de lo ambiental como asunto público (Miñana, Toro y Mahecha, 2012). Según "Su institucionalización y obligatoriedad en los diferentes países son una expresión de que esta problemática es asunto nacional y mundial sobre el que la sociedad en su conjunto -a través de los órganos legislativos- debe ocuparse" (Miñana et al., 2012, p. 1151). De esta manera, en Colombia, a partir de 1994, a través de la Ley 115, decretos 1743 y 1860, se abordan las diferentes problemáticas ambientales a través de estrategias como los proyectos ambientales escolares (Prae) (Miñana et al., 2012), los cuales han ido avanzando frente a su forma de abordaje y se han constituido en una estrategia importante en la educación ambiental.

Para Badillo (2012) en Colombia la necesidad de dimensionar social y políticamente la educación ambiental se ha manifestado en diversas posiciones estatales (a veces, decididas; a veces, totalmente ausentes) y a través de una transición, que inicia desde la década de 1970 con una visión ecologista de la educación; luego, hacia 1980, cuando sobre el desarrollo sostenible se dinamizan otras configuraciones sobre lo ambiental, y continuando en 1990, al constituirse una corriente transversal de los procesos educativos ambientales. (p. 89)

Se debe considerar este como un proceso formativo claramente estratégico, el cual debe direccionarse desde el contexto real del entorno y orientarse considerando las diferentes necesidades ambientales globales y de acuerdo con la evolución de los conocimientos científicos, ofrecer respuestas y propuestas coherentes, estructuradas y efectivas que permitan en la comunidad educativa enfrentarse a un proceso de adaptación y generación de posibles soluciones a dichas necesidades.

Melendro, Murga, Novo y Bautista-Cerro (2008) plantean:

La adquisición de las competencias formativas ha de ser abordada desde una metodología educativa innovadora, que combine de forma equilibrada las necesidades de adquisición y asimilación de conocimientos con su adecuada aplicación a la práctica educativa, y que a su vez proporcione referencias prácticas y experimentadas a la construcción del conocimiento. (p. 22)

Por otro lado, Duarte y Valbuena (2014) mencionan que "la Educación Ambiental en la escuela contemporánea se presenta como un gran desafío en el que resulta importante ubicar a los maestros como agentes capaces de intervenir en los procesos ambientales que poseen 
doi: http://dx.doi.org/10.15359/ree.22-2.5

URL: http://www.una.ac.cr/educare

CORREO: educare@una.cr

las comunidades educativas que integran" (p. 27); es decir, estos procesos deben partir de un desarrollo de apropiación, sensibilización y contextualización de las personas formadoras; pues no se puede pensar que este tema sea abordado por profesorado desconocedor del contexto; en este sentido, este tema en cada establecimiento educativo debe estar direccionado por quienes se han formado para tal fin; sin embargo, es importante anotar la necesidad de no solo pensarse en una educación ambiental orientada a la comunidad educativa, sino también en trascender a una preparación hacia la ciudadanía ambiental, la cual, de acuerdo con González-Gaudiano (2003): "debe estar enmarcada dentro de una política ambiental y cultural; [es decir]; debe [ser] como un proceso donde la formación de ciudadanos fecunda la gestación de relaciones apropiadas entre nosotros y con el medio" (p. 614).

Duarte y Valbuena (2014, citando a González- Gaudiano, 2000) expresan:

En la actualidad en el ámbito escolar muchas prácticas pedagógicas tradicionales mantienen la educación ambiental en una época de resistencia, en la que se piensa que el ambiente forma parte del contenido escolar tan solo por el hecho de hablar de vegetación y fauna. (p. 27)

Es necesario trascender en el contexto y avanzar hacia la búsqueda de una educación para el desarrollo sostenible.

Según García-Esteban y Murga-Menoyo (2015):

A lo largo de su historia, la Organización de las Naciones Unidas ha defendido los valores relacionados con la dignidad humana, las libertades fundamentales, los derechos humanos, la equidad y el cuidado del medio ambiente. El desarrollo sostenible lleva esos valores un paso adelante, ampliándolos más allá de la generación actual a las generaciones futuras. (p. 123).

Gutiérrez y Priotto (2008, p. 533) agregan:

La adopción de una u otra perspectiva se plasma en dos formas también contrapuestas de entender la EADS [educación para el desarrollo sostenible]: como una tecnología social cuyo fin último es [prevenir], minimizar [y controlar] los impactos [ecosistémicos] negativos de la civilización industrial mismos, suscribiendo las "tesis blandas" del desarrollo sostenible; o como una "pedagogía o praxis crítica", cuyos fines y estrategias de acción inspiran proyectos -en ocasiones cargados de contenidos utópicos- de cambio global hacia una civilización "socialmente más justa y ecológicamente sostenible". 
De acuerdo con lo anterior, la educación ambiental es interdisciplinaria, debe contribuir a analizar realidades sociales y naturales, y procurar ser transversal a todas las ramas del conocimiento, pues necesita de la totalidad de las disciplinas y los saberes para su construcción (Ruíz y Pérez, 2014).

Las estrategias implementadas en la comunidad educativa no son de gran utilidad, si no van acompañadas de medidas de gestión eficaces (Benayas, Gutiérrez y Hernández, 2003). Para establecer dichas estrategias se requiere tener conocimiento de la comunidad que se está abordando, así como de las percepciones y comportamientos, pues esto permitirá un acercamiento y definir la forma de abordaje más adecuada para cada tipo de comunidad. En la Fundación Niños de los Andes, sobre la cual se tiene una institución de educación para niños, niñas y adolescentes en condición de vulnerabilidad, se hace necesario plantear estrategias eficientes que permitan mejorar las condiciones ambientales de esta misma.

Para formular la estrategia de educación y capacitación con la comunidad, con respecto al manejo, operación y mantenimiento del sistema de tratamiento que se debería abordar en la Fundación Niños de Los Andes, fue necesario no solo pensar en un proceso de abordaje de la temática concreta, sino también hacer un análisis de cómo las personas participantes de la fundación asimilaban el tema de la educación ambiental y qué tipo de herramientas podrían ser más eficientes en el momento de abordar la sensibilización ambiental. De esta manera, de acuerdo con este análisis, se pretendió conocer bajo qué formas se podrían abordar las problemáticas ambientales con el estudiantado, que fueran de una manera creativa y asimilable para lograr un mejor resultado. Por las actividades que ha desarrollado la Universidad Católica de Manizales en la Fundación Niños de los Andes, en esta investigación no solo se abordó al personal docente de dicha Fundación, sino también al personal docente que orienta la educación ambiental en la Universidad.

\section{Métodos}

La Fundación Niños de los Andes es un albergue para jóvenes. Es una organización sin ánimo de lucro, concebida como fórmula de protección y rehabilitación de un grupo poblacional creciente de niños y niñas de la calle, en condiciones de gran vulnerabilidad a la violación de sus derechos fundamentales. La población infantil atendida tiene entre 6 y 21 años; se les concede alimentación, hospedaje y estudio desde primer grado hasta noveno grado. Las políticas de la fundación se ajustan a los requerimientos del Estado colombiano, motivo por el cual las sedes tienen sus licencias de funcionamiento vigentes. La sede del municipio de Manizales (Caldas, Colombia) está ubicada en el Parque Adolfo Hoyos Ocampo, en la vereda El Arenillo, en la comuna Atardeceres.

La investigación es de tipo mixto, pues tiene tanto características de investigación cualitativa como cuantitativa con un enfoque descriptivo. 
doi: http://dx.doi.org/10.15359/ree.22-2.5

URL: http://www.una.ac.cr/educare

CORREO: educare@una.cr

\section{Muestra}

Se realizaron 95 encuestas en la totalidad del estudiantado de los grados 3 a 9, 11, el muestreo es no probabilístico al 100\% de la población.

Para el abordaje del equipo docente que orienta la educación ambiental, se tuvo en cuenta no solo al personal docente de la Fundación sino los de la Universidad que orientan educación ambiental; en cada institución la totalidad de la muestra fue de 5 docentes.

\section{Técnicas e instrumentos}

Encuesta, donde se consideraron preguntas para el estudiantado tales como: si le han hablado del medio ambiente, si les gusta el tema; si le han llegado a obsequiar revistas o cartillas que hablen del medio ambiente; si reconocen que en la fundación hay problemas ambientales, que tipo de problemas identifican; si utilizan el internet como herramienta para consultar problemas ambientales y soluciones a estos mismos; sus preferencias por el dibujo, en que temáticas; a través de qué medios le gustaría conocer los problemas de contaminación y su solución, entre otras.

Entrevista semiestructurada para la aplicación con el personal docente. Con preguntas relacionadas con lo siguiente: Si se tienen diferentes contextos de aprendizaje y cuál es el que más se utiliza; cómo se aborda el tema ambiental en el estudiantado, tanto en la Universidad como en la Fundación; cómo se aborda las actividades comprensivas con el estudiantado, escenarios colectivos, individuales, etc.; cómo se abordan las actividades investigativas, experimentales y de comunicación; qué tipo de recursos y herramientas se utilizan para el aprendizaje, y cómo se hace el proceso evaluativo

\section{Procedimiento}

La encuesta se basó en preguntas relacionadas con las preferencias que tenía el estudiantado al momento de abordar temáticas ambientales; estas preguntas fueron cerradas. Para el caso de la entrevista al profesorado, las preguntas fueron abiertas.

Las preguntas estuvieron diseñadas para conocer no solo las percepciones que tienen frente al medio ambiente, sino también de sus preferencias para conocer las temáticas ambientales.

Para la sistematización de los datos se usó el software SPSS y, posteriormente, se procedió a realizar el análisis con el fin de determinar las preferencias del estudiantado y así poder formular la estrategia de capacitación dirigida al estudiantado y personas formadoras ambientales. 
Igualmente, se tuvo en cuenta la percepción del personal docente tanto de la Universidad Católica de Manizales como de la Fundación Niños de Los Andes, con el fin de conocer sus posturas sobre la receptividad que ha tenido el estudiantado frente a los temas ambientales; información que se obtuvo a partir de entrevistas semiestructuradas. La finalidad de involucrar al equipo de docentes de la Universidad fue para darle una mayor relevancia metodológica y mayor soporte a la aproximación de la formulación de la estrategia de educación ambiental.

\section{Aproximación a la formulación de la estrategia}

Para la aproximación a la formulación de la estrategia se procedió a utilizar los resultados de la encuesta, y la sistematización de todos los datos, con el propósito de seleccionar la estrategia más llamativa para el estudiantado; una vez que se determinó esta, se buscaron antecedentes de cómo han sido abordadas estas temáticas en otros escenarios para proceder a formular la estrategia propia del proyecto.

\section{Resultados}

\section{Percepción del estudiantado frente a las actividades para el abordaje de la educación ambiental}

En la Figura 1 aparecen los resultados asociados a la percepción del estudiantado frente a las formas de abordaje de los temas de educación ambiental.

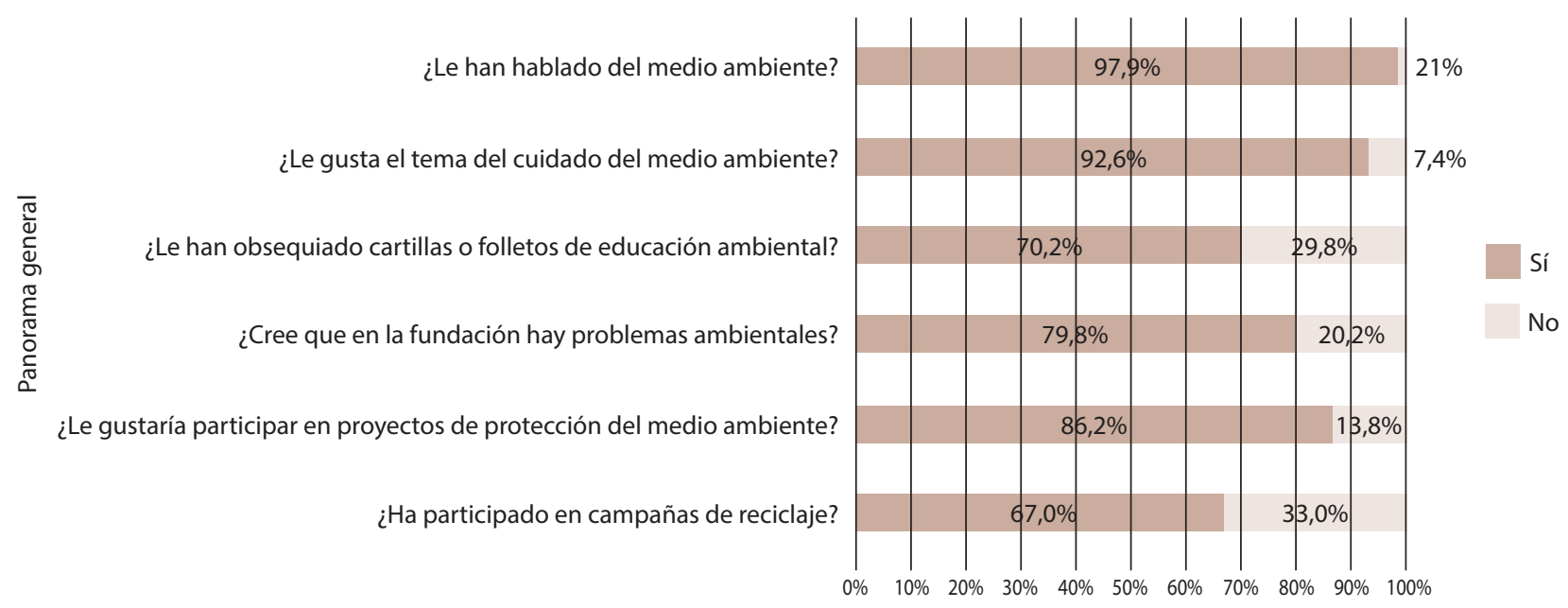

Figura 1: ¿Percepción de la educación ambiental en el estudiantado?

Nota: Propia del proyecto. 
doi: http://dx.doi.org/10.15359/ree.22-2.5

URL: http://www.una.ac.cr/educare

CORREO: educare@una.cr

\section{Abordaje del tema del medio ambiente en el estudiantado}

Como se puede observar, frente a la pregunta sobre si se le ha hablado del tema del medio ambiente, el 97,9\% del estudiantado manifestó que sí les han hablado del tema, a través de diferentes medios; estos temas han sido abordados en diferentes contextos, pero especialmente desde las instituciones educativas.

En la Figura 2 se muestra la preferencia por el medio en que al estudiantado le gusta más que les hablen del medio ambiente.

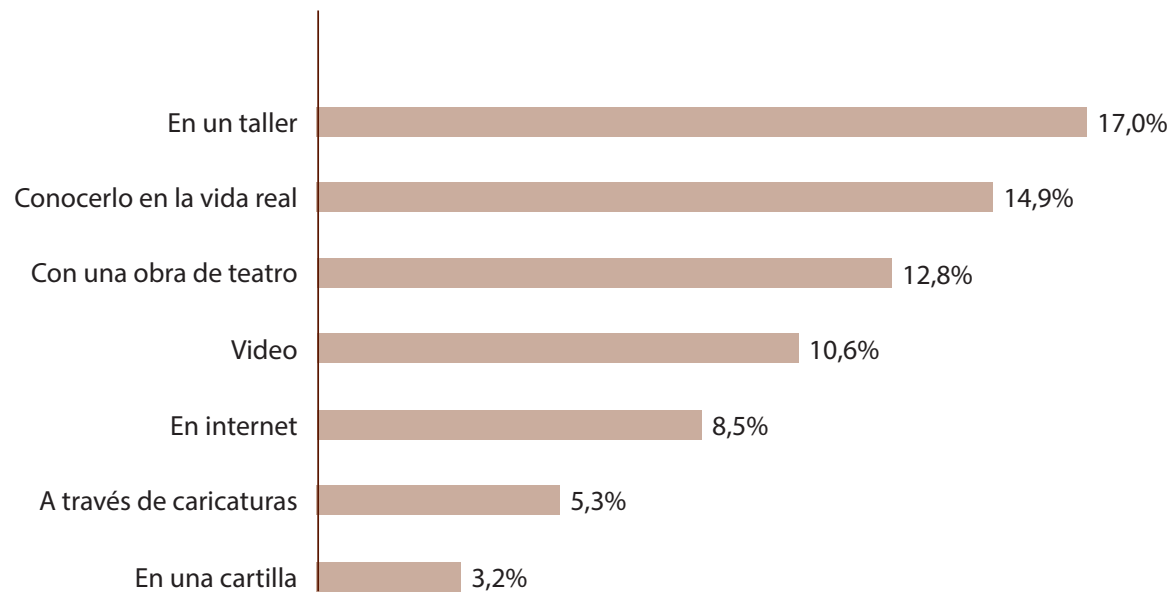

Figura 2: ¿Por qué medio prefiere que le hablen del medio ambiente?

Nota: Propia del proyecto.

El estudiantado prefiere que le hablen del medio ambiente, como se puede apreciar en la Figura 2, a través de un taller -cabe anotar que estos talleres se refieren a las formas prácticas de realizar las actividades-, se presenta un menor porcentaje de preferencia por el uso de las cartillas; puede inferirse, de acuerdo con este resultado, que la mejor forma de abordar la temática ambiental es a través del desarrollo de talleres que sean muy lúdicos y prácticos.

\section{Motivación por el cuidado del medio ambiente}

El 92,6\% del estudiantado manifiesta gusto por el cuidado del medio ambiente, mientras que para el $7,4 \%$ restante el tema le aburre o le es indiferente. Podría inferirse que este porcentaje de estudiantes no ha tenido una motivación suficiente para que muestre un mayor interés por el tema; en este sentido, una buena estrategia de sensibilización podría ser de gran apoyo para su motivación. 


\section{Utilización de herramientas como cartillas o folletos}

Una de las herramientas más abordadas en la educación ambiental para los procesos de sensibilización ha sido la utilización de cartillas y folletos, los cuales han estado direccionadas al conocimiento y protección de los recursos naturales, generalmente de acuerdo con el público objetivo se han trabajado animaciones caricaturescas que permiten generar motivaciones especialmente en la niñez.

Para el caso de estudio, al 70,2\% de estudiantes le han obsequiado cartillas o folletos relacionados con temáticas ambientales, las cuales han sido entregadas a través de campañas o estrategias implementadas en la Fundación para promover la educación ambiental.

Por otro lado, es importante anotar que, de acuerdo con el resultado anterior frente al porcentaje de preferencia por la utilización de las cartillas, la mayoría del estudiantado manifiesta que las ha leído o las ha utilizado como medio de consulta y, en un menor porcentaje, las ha desechado como aparece en la Figura 3.

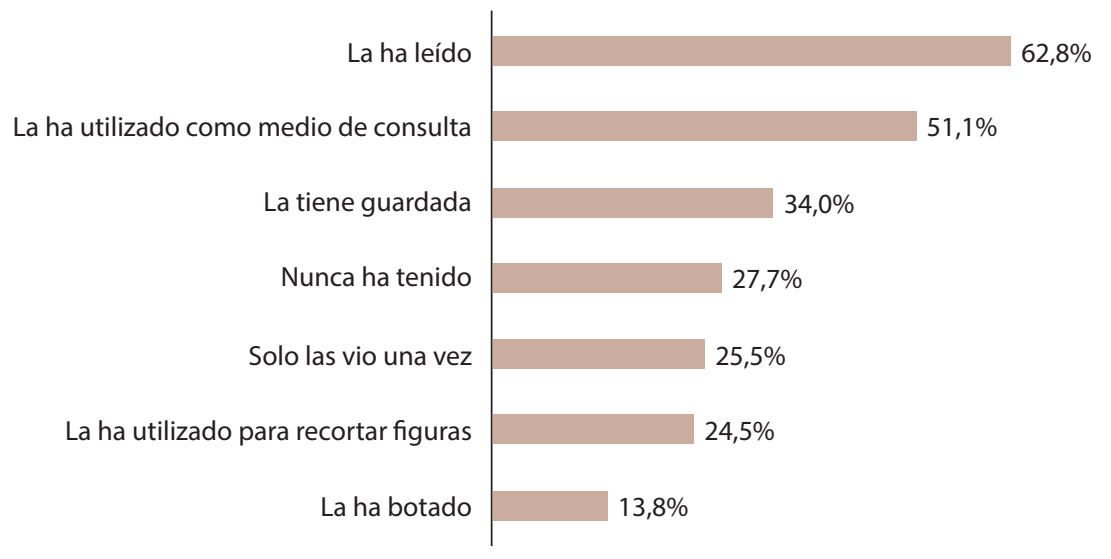

Figura 3: ¿Cuál es el uso que le ha dado a las cartillas que le han obsequiado?

Nota: Propia del proyecto.

\section{Reconocimiento de los problemas ambientales en la Fundación}

Frente a la pregunta sobre si la niñez cree que en la Fundación hay problemas ambientales, más del 70\% argumenta de la existencia de dichos problemas, los cuales de acuerdo con lo que manifiestan, están relacionados con aguas negras y con el manejo de residuos principalmente. Puede inferirse que hay un reconocimiento de las problemáticas existentes en la fundación por parte del estudiantado, lo que es una ventaja a la hora de tomar medidas frente a los asuntos ambientales más representativos, igualmente puede ser el punto de partida para generar una estrategia de capacitación tanto para el estudiantado como para docentes de la Institución. 
doi: http://dx.doi.org/10.15359/ree.22-2.5

URL: http://www.una.ac.cr/educare

CORREO: educare@una.cr

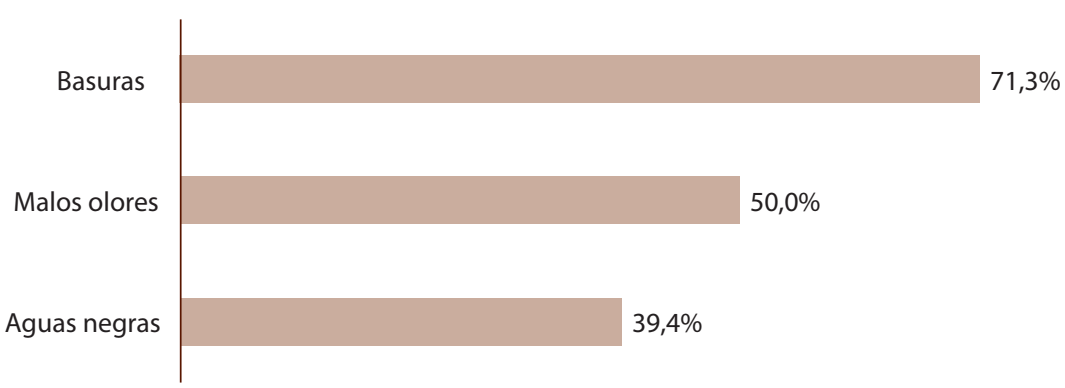

Figura 4: ¿Que tipo de problemas ambientales identificas en la Fundación?

Nota: Propia del proyecto.

De acuerdo con la Figura 4, el problema ambiental que más percibe el estudiantado es el de mal manejo de residuos; ocupa el segundo lugar el relacionado con malos olores, los cuales se dan principalmente por el mal manejo de aguas negras.

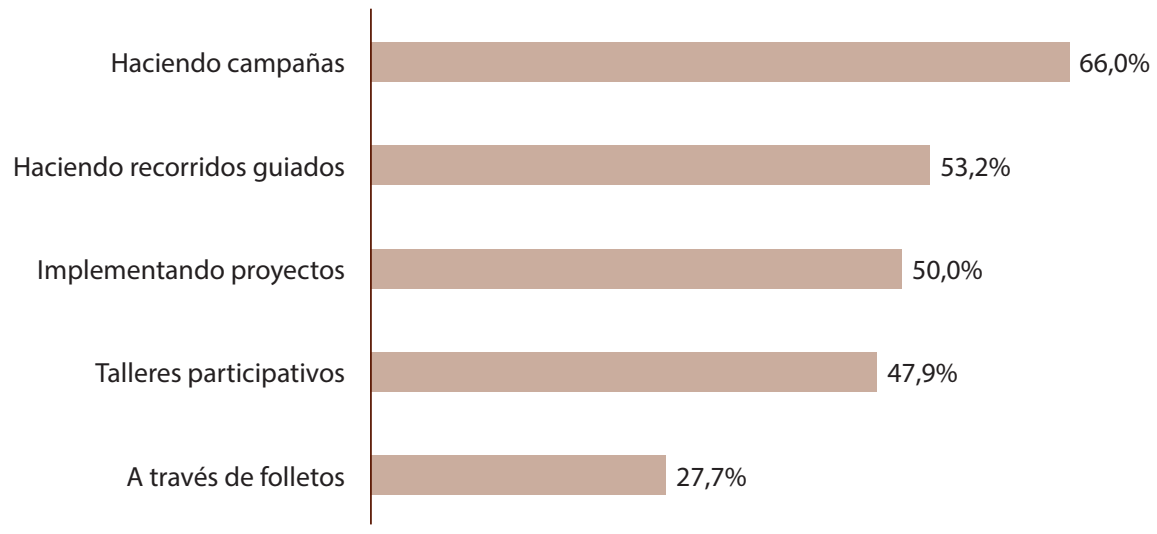

Figura 5: ¿De qué forma prefiere conocer y controlar los problemas ambientales?

Nota: Propia del proyecto.

En la Figura 5 se muestran las diferentes alternativas que el estudiantado manifiesta que se pueden emplear para enfrentar los problemas ambientales en la Fundación. Reconoce las campañas como principal forma de controlar dichos problemas, seguido de recorridos guiados que permitan no solo conocer de primera mano las principales problemáticas, sino también interiorizar una forma más práctica de sensibilización ambiental; los folletos o cartillas siguen siendo para el estudiantado la manera de menor preferencia para controlar o conocer las formas de abordaje de las soluciones ambientales.

\section{Contaminación del agua}

Frente al conocimiento sobre la contaminación del agua, el estudiantado tiene preferencia por ver los ejemplos directamente en campo, seguido de la realización de talleres, las cartillas 
doi: http://dx.doi.org/10.15359/ree.22-2.5

URL: http://www.una.ac.cr/educare

siguen estado en menor porcentaje de preferencia. El que tiene menos preferencia o mención de respuesta es el programa de televisión, como aparece en Figura 6.

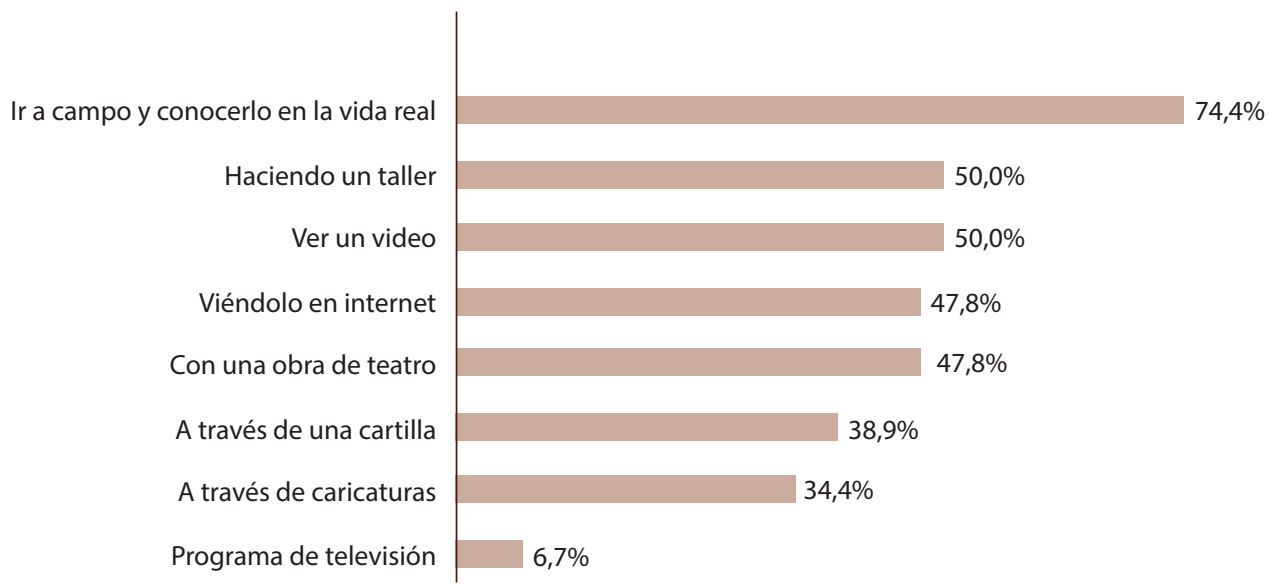

Figura 6: ¿Por qué método prefiere conocer los problemas ambientales asociados a la contaminación del agua?

Nota: Propia del proyecto.

Con la pregunta anterior se indagó, sobre las preferencias para conocer esas soluciones ambientales asociadas a la contaminación del recurso hídrico. Se obtuvo el resultado que aparece en la Figura 7.

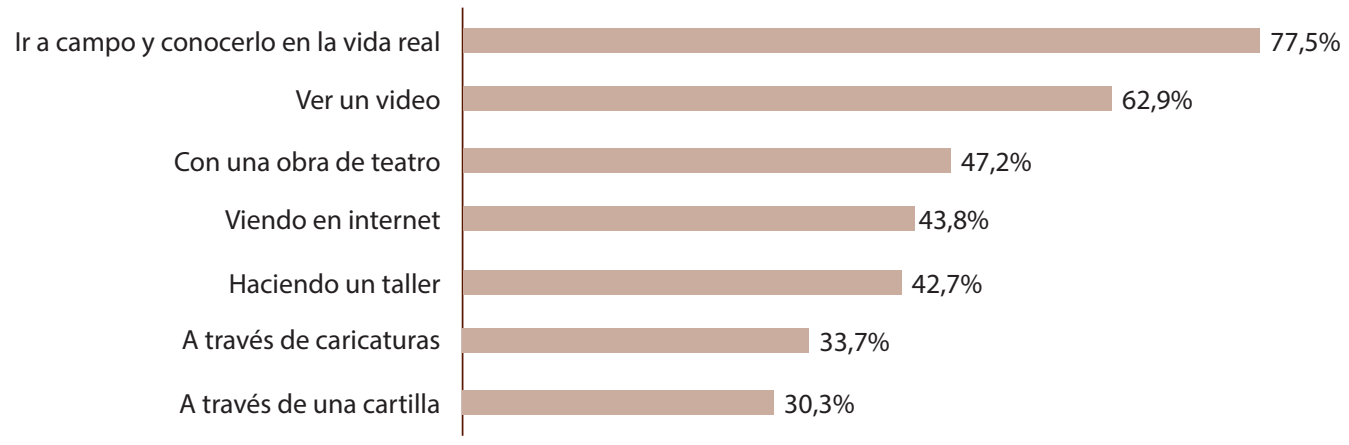

Figura 7: ¿A través de qué medio prefiere conocer las soluciones a las problemáticas asociadas al recurso hídrico?

Nota: Propia del proyecto. 
doi: http://dx.doi.org/10.15359/ree.22-2.5

URL: http://www.una.ac.cr/educare

CORREO: educare@una.cr

Ir a campo y hacer un procedimiento o taller práctico sigue siendo la mejor herramienta para conocer las soluciones a los problemas ambientales, también manifiestan que podría ser a través de un recurso didáctico como un video.

Una de las preguntas realizadas correspondió a la frecuencia con que el estudiantado mira el internet para conocer problemas ambientales, donde más del $40 \%$ manifiesta que nunca miran el internet para esto, como aparece en la Figura 8.

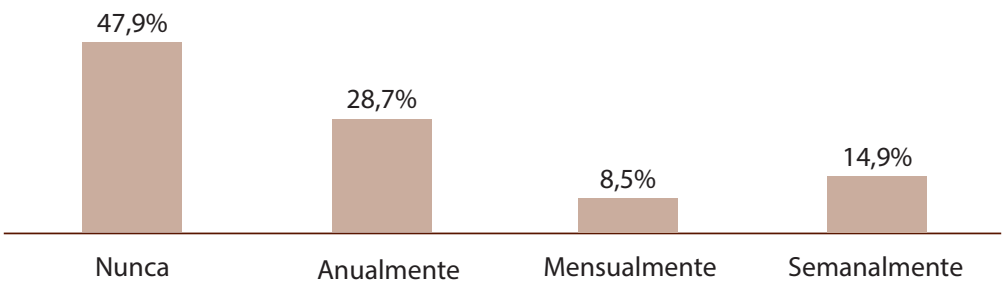

Figura 8: Porcentaje de la frecuencia para utilizar el internet y consultar problemas ambientales.

Nota: Propia del proyecto.

Otro de los aspectos importantes y que han sido motivadores para el estudiantado es el trabajo a través de dibujos, pues esta se considera una forma tradicional de exponer sus sentimientos frente a sus sueños o visiones del entorno. Ante la pregunta si les gusta dibujar, se obtuvo el resultado de la Figura 9.

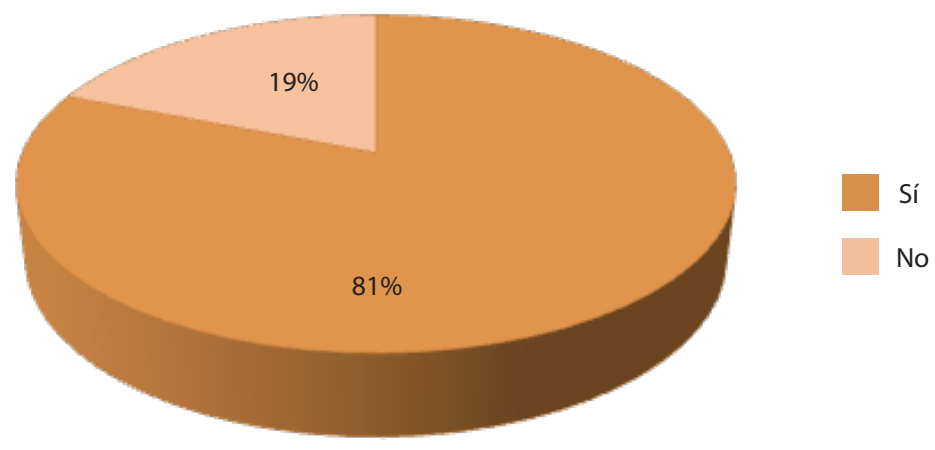

Figura 9: Respuestas a la pregunta si al estudiantado le gusta dibujar.

Nota: Propia del proyecto. 
El $81 \%$ del estudiantado manifiesta que si les gusta dibujar y sus preferencias están básicamente en dibujar paisajes, como aparece en la Figura 10.

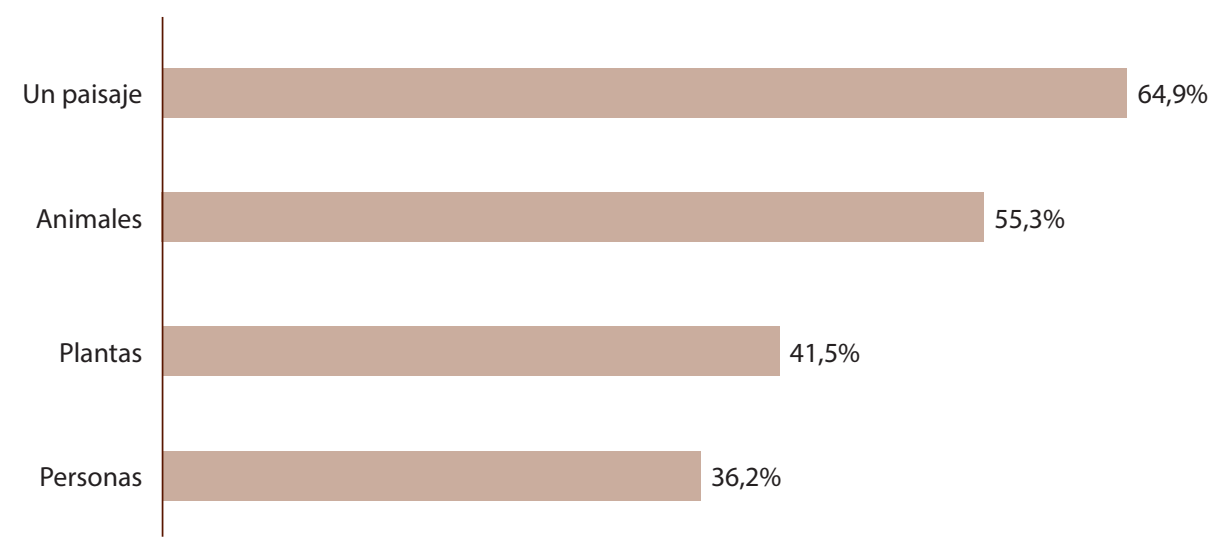

Figura 10: Preferencia de las temáticas en el momento de dibujar.

Nota: Propia del proyecto.

\section{Participación en proyecto sobre protección del medio ambiente}

Frente a esta pregunta, el 86,2\% del estudiantado manifiesta que le gustaría participar de este tipo de proyectos; de lo que se puede inferir que hay un alto porcentaje de interés por parte del estudiantado en apropiarse y ser parte de proyectos que tienden al mejoramiento de su entorno.

\section{Participación en campañas de reciclaje}

A esta pregunta, el $67 \%$ del estudiantado manifiesta que ha participado de este tipo de campañas, y el 33\% restante no conoce el tema; lo que significa que sería muy importante implementar este tipo de estrategias de sensibilización en la Fundación.

El estudiantado manifiesta, como aparece en la Figura 11, que le gustaría trabajar el reciclaje preferentemente de una manera práctica, las cartillas se encuentran en menor porcentaje de preferencia igual que la realización de caricaturas.

La construcción de videos es otra alternativa que puede generar impacto en la niñez, especialmente si ellos y ellas participan de la construcción de esos mismos.

Las cartillas y folletos son las estrategias que menos llama la atención de la niñez para conocer tanto los problemas ambientales como su forma de abordaje. 
doi: http://dx.doi.org/10.15359/ree.22-2.5

URL: http://www.una.ac.cr/educare

CORREO: educare@una.cr

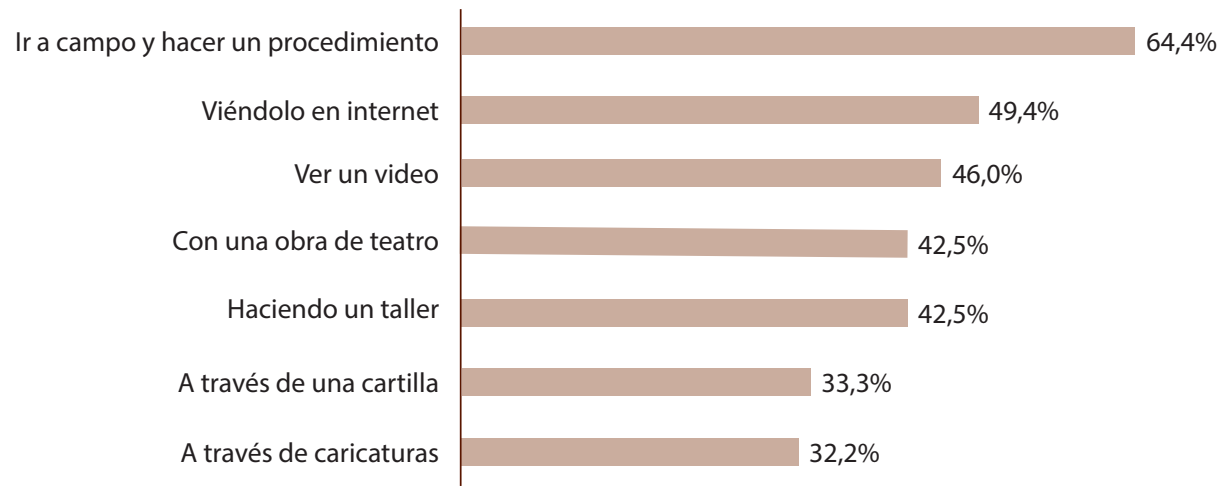

Figura 11: ¿Cómo le gustaría abordar el tema del reciclaje?

Nota: Propia del proyecto.

Estrategias de aprendizaje utilizadas por el personal docente para el abordaje de la educación ambiental, tanto en la Fundación Niños de los Andes como en la Universidad Católica de Manizales

Para el abordaje de las estrategias de aprendizaje se tuvieron en cuenta cinco categorías, las cuales se consideran en las instituciones estudiadas y están referenciadas en el estudio realizado por Marcelo et al. (2014) titulado Las actividades de aprendizaje en la enseñanza universitaria: ¿Hacia un aprendizaje autónomo de los alumnos? Los siguientes resultados se encuentran unificados de acuerdo con las respuestas obtenidas por el personal docente de cada una de las instituciones:

\section{El contexto de aprendizaje}

Referente al contexto de aprendizaje, todo el personal docente de la Universidad Católica de Manizales manifiesta que se tienen en cuenta diferentes contextos, sin embargo, el aula de clase es el que se utiliza con más frecuencia. La educación ambiental es abordada como una asignatura obligatoria para todas carreras de pregrado, pero, desafortunadamente, se hace a través de un programa académico de asignatura (PAA) generalizado, sin considerar particularidades propias de cada carrera. Esto ha podido generar algún desinterés en el estudiantado por considerar que son temas ajenos a su objeto de estudio.

Otro contexto de trabajo ha sido en trabajo de campo, aunque este se ha realizado en menor proporción, con excepción del estudiantado de Ingeniería Ambiental que, por su formación, desarrolla diferentes actividades en un contexto real en campo; la percepción que el equipo docente tiene es que este contexto de aprendizaje facilita que el estudiantado pueda interactuar directamente con el ambiente y se puedan visualizar con mayor precisión las problemáticas o soluciones ambientales de las que se habla en clase. 
Ahora bien, en cuanto a la Fundación Niños de Los Andes, el personal docente manifiesta que el componente ambiental es orientado desde asignaturas como las ciencias naturales y que el tema no se aborda de manera particularizada, sino que se promociona a partir de la realización de campañas específicas que contribuyan a generar sensibilización en el estudiantado, las cuales, en su mayoría, terminan siendo olvidadas por la niñez.

Frente al contexto de aprendizaje, este se ha trabajado tanto en el aula como en campo, teniendo una mayor aceptación el trabajo en campo.

Igualmente se tienen en cuenta, en menor proporción, otro tipo de contextos como los virtuales y la biblioteca, considerados en la Universidad, pero que no se tienen en cuenta en la fundación, ya que no se cuenta con este tipo de espacios.

\section{Tipo de actividad}

Las actividades abordadas: Actividades comprensivas, actividades investigativas, actividades experimentales, actividades de comunicación.

Dentro de las actividades comprensivas se consideran aquellos espacios donde, a partir de lectura de documentos relacionados con aspectos ambientales, se realizan análisis y escritos; también en este tipo de actividades se consideran la visualización y análisis de los diferentes videos relacionados con temas de sensibilización ambiental, muchos de ellos son documentales.

Este tipo de tareas comprensivas se abordan de forma preferiblemente colectiva para tener la posibilidad de que cada estudiante manifieste sus interpretaciones de la comprensión que tuvo sobre la temática. Este tipo de actividades se logra, en mayor proporción, en el escenario de la Universidad y en menor proporción en la Fundación.

Respecto a las actividades investigativas, se consideran aquellas que motivan al estudiantado a realizar consultas, a indagar tanto acerca de problemáticas ambientales como a posibles soluciones; se motiva a hacerse preguntas y buscar respuestas; todo el personal docente universitario encuestado manifiesta que estas se desarrollan en mayor proporción en la Universidad y se hace generalmente de manera individual, pues se pretende que el estudiantado sienta una postura crítica frente a lo investigado; es importante destacar la modalidad de los proyectos integradores que hacen parte de la investigación formativa, donde se permite la articulación y transversalización de los temas ambientales con otros temas afines o no.

Dentro de las actividades experimentales se destacan aquellas relacionadas con trabajos de campo o procedimientos de laboratorio, las cuales, a pesar de ser de mayor preferencia para el estudiantado, no son muy promovidas por el personal docente; con excepción del programa de ingeniería ambiental, el cual, por su condición de programa académico, lo requiere necesariamente. 
doi: http://dx.doi.org/10.15359/ree.22-2.5

URL: http://www.una.ac.cr/educare

CORREO: educare@una.cr

En la Fundación Niños de Los Andes este tipo de estrategias no se lleva a la clase con frecuencia, salvo por algunas actividades lideradas por la Universidad Católica de Manizales, donde se han realizado actividades prácticas relacionadas con procesos de descontaminación y tratamiento de aguas residuales, en cuya participación estudiantil se ha evidenciado una buena acogida.

Dentro de las actividades de comunicación se han manejado estrategias relacionadas con las exposiciones de temáticas ambientales, en las cuales el estudiantado plantea sus posturas frente a las problemáticas locales, nacionales $y$, en algunas ocasiones, internacionales; se plantea el debate como un mecanismo de participación colectiva que se hace interesante para el estudiantado.

\section{Los recursos y herramientas}

En cuanto a los recursos y herramientas para el abordaje de la educación ambiental, el personal docente de la Universidad entrevistado manifiesta que se utilizan pizarras físicas la mayoría de las veces y otras virtuales a través de la plataforma Modle, sobre la cual se pueden visualizar diferentes herramientas como videos y otros recursos como foros de discusión, tareas, entre otros; para el caso de la Fundación Niños de Los Andes, las herramientas virtuales son limitadas, pues no se cuenta con una infraestructura adecuada que permita su uso.

\section{La evaluación}

La evaluación es el momento en el que el personal docente comprueba las competencias que el estudiantado ha adquirido a través de las actividades realizadas en los procesos de educación ambiental. La Universidad tiene tres momentos en cada semestre para dicha evaluación, la cual es distribuida por cortes.

Dentro de cada corte se realizan talleres evaluativos, evaluaciones escritas, se evalúan las exposiciones del estudiantado. En la evaluación la única finalidad es interpretar el cumplimento de las competencias propuestas por cada docente para la comprensión de la educación ambiental; por otro lado, el estudiantado no es el único que es evaluado, también ocurre de manera viceversa, pues el personal docente es evaluado por el estudiantado, lo que le permite al personal educativo identificar las posibilidades de mejora en su desempeño.

Para el caso de la Fundación, no existe la evaluación.

\section{Fase propositiva}

En esta fase se hace una aproximación a la estrategia de capacitación en educación ambiental, partiendo del conocimiento previo en la fase diagnóstica; en este sentido, se consideran los siguientes aspectos para la construcción de esta: 


\section{Caracterización de la estrategia}

Rodríguez et al. (2011), mencionan:

Una estrategia de educación ambiental es un conjunto de acciones coherentemente diseñadas en programas, orientadas a medio plazo (porque así lo requieren los conflictos ambientales) y que aglutina esfuerzos de un conjunto amplio de agentes sociales (gestores, educadores, políticos, ciudadanos y asociaciones) en aras de mejorar o cambiar una realidad socioambiental en un contexto geográfico concreto. (p. 5)

\section{Principios de la estrategia}

La estrategia de educación ambiental para la Fundación Niños de Los Andes debe estar orientada, principalmente, desde los siguientes principios:

Integralidad: la educación ambiental en la Fundación debe concebirse desde todos los contextos, principalmente el humano; debe partir de un reconocimiento y apropiación de la relación niñez-ambiente.

Práctica: este principio está enfocado principalmente por los resultados obtenidos en la fase diagnóstica, donde la niñez manifiesta que su mejor forma de adquirir motivación y apropiación de los procesos es a través de ejercicios prácticos que le permitan tener un acercamiento más concreto con la realidad que están abordando.

Reconocimiento: este principio está basado en la necesidad de que el estudiantado reconozca su entorno y aprenda a identificar y reconocer tanto las fortalezas como las debilidades ambientales que lo rodean.

Coherencia: la coherencia se refiere a la necesidad de establecer estrategias que sean acordes con sus preferencias de aprendizaje; de acuerdo con el diagnóstico, las preferencias están dirigidas a trabajos prácticos, talleres y campañas.

Transversalidad: este principio tiene que ver con que la estrategia no puede desarrollarse como una actividad más de la institución, sino que debe articularse y permear en todos los procesos sustantivos que se desarrollen dentro de ella.

\section{Planeación estratégica}

De acuerdo con el diagnóstico, se proponen los siguientes programas para desarrollar en la Fundación Niños de Los Andes. 
doi: http://dx.doi.org/10.15359/ree.22-2.5

URL: http://www.una.ac.cr/educare

CORREO: educare@una.cr

\section{Programa de embellecimiento}

La Universidad Católica de Manizales ha venido desarrollando un proyecto para la construcción del sendero ecológico del cual ha participado toda la comunidad educativa; el estudiantado ha participado de las diferentes actividades y se la logrado el embellecimiento de la institución.

Se plantea un proyecto de fortalecimiento de un vivero ornamental con el fin de generar en toda la institución una mejor imagen, así como la restauración de espacios naturales.

Se plantea una propuesta de señalización de espacios ambientalmente importantes.

\section{Programa de conmemoración de fechas ambientales}

El objetivo de este programa es sensibilizar al estudiantado a través de campañas y procesos prácticos a través de las celebraciones de los días conmemorables del medio ambiente.

Frente a los resultados obtenidos en la fase diagnóstica, la participación de la niñez en los procesos es un aspecto importante para la sensibilización. En este programa se plantea la realización de diferentes campañas con el fin de conmemorar fechas ambientales importantes. En Colombia se celebran, entre otras, las siguientes fechas:

- Día mundial del medio ambiente

- Día mundial del agua

- El día de la tierra

- Día de concientización respecto al ruido

- Día internacional de las aves

- Día mundial de lucha contra la desertificación y la sequía

- Día mundial del hábitat

- Día del suelo

- Día internacional de la diversidad biológica

\section{Programa de capacitación}

El programa de capacitación estará dirigido a toda la comunidad educativa; su objetivo es crear escenarios de entendimiento y apropiación por el entorno.

Este programa buscará que la comunidad educativa reconozca sus problemas ambientales y, a partir de allí, tenga la capacidad de realizar intervenciones para controlar y mitigar los impactos ambientales. 
Para esta propuesta se proponen principalmente dos procesos de capacitación fuertes: uno dirigido hacia un programa de control de la contaminación del agua, y otro hacia un programa de reciclaje y manejo de residuos.

\section{Control de la contaminación del agua}

Para este programa, la Universidad Católica ha venido desarrollando el proyecto para el tratamiento de las aguas residuales.

Con base en esta planeación estratégica, se plantea que se desarrollen talleres de sensibilización al estudiantado con el fin de conocer los diferentes métodos de abordaje de la contaminación hídrica.

Se plantea un ejemplo de una ficha técnica (ver Figura 12) para realización de los talleres.

Objetivo de los talleres:

Sensibilizar al estudiantado en el reconocimiento de la contaminación por el mal manejo de los residuos sólidos, y la búsqueda de alternativas para su control y disminución de impacto ambiental

Proceso metodológico:

De acuerdo con los resultados de la fase diagnóstica, los talleres deberán realizarse de manera práctica:

- Presentación de los participantes (se propone un juego para esta presentación considerando un animal con el que se identifiquen).

- Contextualización teórica de las problemáticas ambientales

- Recorridos por la institución para la identificación de las problemáticas

- Discusión de estas problemáticas desde sus puntos de vista: aquí se pretende generar una postura crítica por parte del estudiantado.

- Propuestas de soluciones a dichas problemáticas en plenaria.

- Desarrollo del trabajo práctico para la resolución de la problemática: para el caso de la contaminación hídrica se hará un modelo de tratamiento de aguas residuales a partir de humedales artificiales.

- El estudiantado deberá hacer un cronograma para determinar la forma de seguimiento y evolución del modelo montado.

- En cada taller se establecerán las conclusiones, recomendaciones y compromisos.

Método de evaluación

Se harán evaluaciones colectivas en forma de test, con participación del estudiantado donde se generen espacios de discusión de los conceptos aprendidos.

Figura 12: Ficha técnica.

Nota: Elaboración propia. 
doi: http://dx.doi.org/10.15359/ree.22-2.5

URL: http://www.una.ac.cr/educare

CORREO: educare@una.cr

\section{Conclusiones}

En conclusión, para desarrollar una herramienta de capacitación y abordaje de las temáticas de educación ambiental en la Fundación Niños de Los Andes, se debe partir de talleres vivenciales, es decir, que se puedan hacer talleres con recorridos guiados donde el estudiantado pueda dar cuenta de las problemáticas reales ambientales y de las posibilidades de abordaje para su control y mitigación.

Las estrategias para la educación ambiental deben estar considerar, como foco, las particularidades de los diferentes grupos de la comunidad educativa, de esta manera es importante conocer su cultura, percepciones y gustos por las formas de abordaje de diferentes temáticas.

De las herramientas analizadas en el presente proyecto de investigación, la utilización de las cartillas es la menos indicada, pues el estudiantado pierde el interés por estas mismas, lo cual las convierte, en muchas ocasiones, en material de reciclaje o en material para recortar figuras.

Los talleres prácticos se consideran, de acuerdo con la investigación, las mejores herramientas de apropiación no solo de las problemáticas ambientales presentes, sino también de las formas de abordaje.

La realización de videos puede ser una buena herramienta o mediación pedagógica, ya que este requiere mayor contribución y permite que, al verse en el documental final, sus participantes tengan más sentido de pertenencia por su entorno.

Sobre las formas de abordaje que se están haciendo para sensibilizar en los procesos de educación ambiental, se puede concluir que es importante realizar replanteamientos que conlleven a un mayor interés por parte del estudiantado en los temas ambientales.

Los procesos evaluativos en el estudiantado deben contener más actividades, pues estas se restringen a talleres y evaluaciones concretas; se puede inferir que la evaluación en trabajos de campo podría tener una mayor aceptación por parte del estudiantado; en la medida en que exista una mayor receptividad en el aprendizaje, también habrá una mayor respuesta en el momento evaluativo.

\section{Referencias}

Badillo, M. E. (2012). Política de educación ambiental en Colombia, 2002-2010. Revista de Investigación Agraria y Ambiental, 3(1), 89-96. doi: https://doi.org/10.22490/21456453.948

Benayas, J., Gutiérrez, J. y Hernández, N. (2003). La investigación en educación ambiental en España. Madrid: Ministerio de Medio Ambiente. Secretaría General de Medio Ambiente. Recuperado de http://www.mapama.gob.es/es/ceneam/recursos/documentos/investigacion-educacionambiental-espana tcm7-13540.pdf 
Duarte, J. J. y Valbuena, E. O. (2014). Referentes de la formación de profesores en educación ambiental. Revisión de antecedentes 2000-2012. Uni-pluri/versidad, 14(2), 27-36. Recuperado de http://aprendeenlinea.udea.edu.co/revistas/index.php/unip/article/ view/20054/16944

García-Esteban, F. E. y Murga-Menoyo, M. Á. (2015). El profesorado de educación infantil ante el desarrollo sostenible: Necesidades formativas. Enseñanza \& Teaching, 33(1), 121-142. doi: https://doi.org/10.14201/et2015331121142

González-Gaudiano, E. J. (2003). Educación para la ciudadanía ambiental. Interciencia, 28(10), 611-615. Recuperado de http://www.redalyc.org/articulo.oa?id=33908509

Gutiérrez, J. y Priotto, G. (2008). Estudio de caso: Sobre un modelo latinoamericano de desarrollo curricular descentralizado en educación ambiental para la sustentabilidad. Revista Mexicana de Investigación Educativa, 13(37), 529-571. Recuperado de http://www.scielo. org.mx/pdf/rmie/v13n37/v13n37a7.pdf

Marcelo, C., Yot, C., Mayor, C., Sánchez, M., Murillo, P., Rodríguez, J. M. y Pardo, A. (2014. Las actividades de aprendizaje en la enseñanza universitaria: ¿Hacia un aprendizaje autónomo de los alumnos? Revista de Educación, 363, 334-359. doi: 10-4438/1988-592X-RE-2012-363-191

Melendro, M., Murga, M. Á., Novo, M. y Bautista-Cerro, M. (2008). Estrategias formativas innovadoras en educación ambiental y para el desarrollo sostenible. Revista lberoamericana de Educación a Distancia, 11(2), 15-39. Recuperado de http://revistas.uned.es/index.php/ ried/article/view/929/850

Miñana, C., Toro, C y Mahecha, A. M. (2012). Construcción de lo público en la escuela: Una mirada desde dos experiencias de educación ambiental en Colombia. Revista Mexicana De Investigación Educativa, 17(55), 1147-1171. Recuperado de http://www.redalyc.org/ articulo.oa?id=14024273007

Rodríguez, L. A., Borroto, M., Gutiérrez, I., Talabera, Y., Quesada, M. y Nuñez, A. (2011). Estrategia para la educación ambiental en comunidades cubanas. Revista Electrónica de Medio Ambiente, 10, 1-12 doi: https://doi.org/10.5209/rev MARE.2011.n10.15851

Ruíz, M. R. y Pérez, E. S. (2014). Educación ambiental en niños y niñas de instituciones educativas oficiales del distrito de Santa Marta. Zona Próxima, 21, 52-64. Recuperado de http://www. redalyc.org/articulo.oa?id $=85332835005$ 